\title{
Mosquito Abatement, Crop Production and Mining Practices Monitoring for the Control of Mosquito-Borne Infectious Diseases
}

Teresa L McKee*

ICP, JD, 14916 Sundial Place, Lakewood Ranch, FL 342020, USA

\begin{abstract}
There is a plethora of articles and studies regarding the emerging and rapidly spreading mosquito borne Zika virus vectored by the Aedes egyptii mosquito. This article focuses on the methods used for mosquito control to reduce the the spread of mosquito borne viruses such as dengue, malaria and Zika virus with speculation regarding the origin and spread of the Zika virus. Other sources of what may have caused the same results as the Zika virus are explored, i.e., what chemicals in the water or crops may have polluted the water or infected the mosquitos through cross-transmission from crops to mosquitoes to simian species to humans that may result in deformities and neurological damage?
\end{abstract}

\section{Introduction}

The Zika virus emerged in Brazil in 2015 with a significant number (over 4,000) [1] of women birthing babies with deformed, small heads and neurological damage. The virus has spread to 22 countries throughout the Americas and the Middle East. It is conjectured that the development of the Olympic facilities in Brazil played a part in this phenomenon since slum and other properties clearance occurred to create the spaces for the stadiums that were constructed in Rio de Janeiro and Sao Paulo, Brazil in preparation for the summer 2016 Olympics. Since the Aedes egyptii mosquito bites during the day and resides in dark places at night, one might assume that workers doing the clearing were bitten by Zika virus carrying mosquitos. These workers subsequently infected their wives and girlfriends through semen, mucus and urine contact. The virus may have entered Brazil for athletic competitions i.e., world games and soccer, from unknowing Zika virus carriers from Africa and the middle East and elsewhere who may have been bitten in Brazil and the mosquito's infected saliva was then transferred to Brazilians when they were bitten afterwards.

\section{Understanding the Zika Virus Progression and other Theories}

A virus is a particle in the blood and in the saliva of a mosquito that once injected into a human by a mosquito bite can travel interstitially to all the bodily fluids i.e., blood, mucus, semen and urine. The female can be infected with the virus by having sex with a male that has been bitten by a mosquito and thus has the virus in his semen or other bodily fluid. A developing foetus can be damaged by the Zika virus via the umbilical cord directly from the mother's blood or through interstitial travel into the uterus of the pregnant female [2]. A mosquito is the vector by which the virus is injected into the human. The mosquito is a receptacle of the virus tainted fluid having bitten either a human who is already infected or an animal (such as a monkey). Another theory on the origin of the birth defects is that the mosquito may be spawned (bred) in contaminated water that infects the mosquito's bodily fluids and resides in the saliva as the mosquito is developing. Another chain of infection transmission scenario is from the simian (apes and monkeys) to mosquito to human via the mosquito bite though there is speculation that the pesticide used in Argentina and Brazil containing Pyriproxyfen actually may cause the microcephaly and nerve damage through breathing in the pesticide or from skin contact with this pesticide. The crop pyrethrum which is the base from which pyriproxyfen and other pyrethrins are manufactured will be discussed later as a source of potential birth defects from the chrysanthemum flower to the simian population via the bee sting and then to the mosquito to the human from the simian population referred to as a zoonotic transfer. An example of zoonotic transfer is the HIV/ AIDS virus which develops in humans after consuming the meat of the simian population. "Bush meat" is a staple in some parts of Africa.

\section{Why is Mosquito Abatement Important?}

Since mosquitoes are the main vector of the Zika virus, eradication is imperative. Twice daily use of repellants, long sleeves and long pants, screens, air conditioning and no standing water in outdoor areas are highly recommended [3]. Mosquito abatement should be aggressively pursued. Some have suggested "guerilla tactics" to go house to house especially in older neighborhoods to remove standing water and spray pesticides to remove breeding areas and to achieve mosquito reduction.

\section{Issues of the Origin of the Zika Virus in Africa and the Relationship to Pyrethrum Cultivation}

The issue of the use of pyreprofyxen in Argentina and Brazil is speculative- scientific research needs to be conducted to determine the correlation of use of this pesticide and the incidence of the Zika virus effects of small heads and neurological damage. Since it has been suggested that this insecticide causes the deformities and neurological damage to newborns, it is recommended that the manufacture of this insecticide be investigated. Pyrethrum is the natural base for the manmade pyreprofyxen. Pyrethrum is extracted from the chrysanthemum flower and is grown in Uganda to produce the pyrethrin insecticides such as pyreprofyxen. And, coincidentally, the Zika Forest near Kampala, Uganda, is thought to be the source of the Zika virus. So, what are the links to the Zika forest and the pyrethrum fields? The mountain gorilla and a myriad of monkey species live in the forests of Uganda and nearby Rwanda. Could they have been infected, bitten and thus the zoonotic transfer occurs with the infected person is bitten or stung and then travels abroad and so we have pregnancies resulting in babies with deformed heads and neurological damage?

*Corresponding author: Teresa L McKee, AICP, JD, 14916 Sundial Place, Lakewood Ranch, FL 342020, USA, Tel: 19417534704; E-mail: TisaL@aol.com

Received March 15, 2016; Accepted June 27, 2016; Published June 30, 2016

Citation: McKee TL (2016) Mosquito Abatement, Crop Production and Mining Practices Monitoring for the Control of Mosquito-Borne Infectious Diseases. J Environ Anal Toxicol 6: 383. doi:10.4172/2161-0525.1000383

Copyright: ( 2016 McKee TL. This is an open-access article distributed under the terms of the Creative Commons Attribution License, which permits unrestricted use, distribution, and reproduction in any medium, provided the original author and source are credited. 
Further research has illuminated the cross-transmission opportunities near the Zika forest in Uganda where the virus and other viruses are being studied. Thus, there may be a connection between the pyrethrin fields and the zoonotic transfer from simians to humans as more travelers attempt sightings and then return to their home countries. What is being transferred? A mutated cell? That is for researchers to discover. This needs to be further investigated as does the possibility that breathing in pyriprofyxen or having skin contact with pyreprofyxen insecticide is somehow responsible for the deformities and health issues of the recent births in Brazil.

\section{Further Discussion of other Sources of the Abnormal Birth Defects and Biodiversity Issues}

Is there a connection between polluted water and birth defects? Could the excessive pollutant load be in waterways as a result of overload of mercury and other heavy metals such as lead where mosquitoes breed thus some adjustment in the total maximum daily load (tmdl) of agricultural and industrial pollutants in Africa and South America be recommended? Should the transboundary movement of the water and wildlife species be monitored or controlled more than current legal agreements and sightings? How can the biodiversity of the gorilla habitat be protected while at the same time getting a handle on disease and contaminant origin and transmission for the gorilla's future as well as for the protection of the human population? Poaching and dismembering gorillas for their body parts makes this a dangerous undertaking. Locating young gorillas sold to zoos or for private pets might be a source of blood and bodily fluid samples to determine their virus carrier profile or mutated cell profile that can be transferred zoonotically.

Risk assessment is recommended for biodiversity protection when evaluating tmdl's, point and non -point pollutant loads and agricultural and industrial applications i.e., pesticides and mercury use [4]. The latter is monitored as a result of the Basel convention and the Minimata conventions [5]. Mercury is one of the most damaging heavy metals to waterway flora and fauna, particularly fish and if concentrations are high might be a mosquito larva contaminant that could lead to in utero damage to the developing fetus if it is residual in the mosquito's saliva when born and transferred to the simian and then cross-transmission from the simian to human and across continents.

There may be a connection between polluted water and birth anomalies. Mercury used in artisanal gold mining in Brazil [6] and Peru has spilled into the Amazon river and its tributaries. Effluent discharge of all mining and manufacturing in Brazil and Peru in South America and in Uganda and Rwanda on the African continent near the forests where mountain gorillas reside should be monitored. These biodiverse "hotspots" of the globe should be evaluated for water quality and heavy metal contaminants particularly to aid in establishing allowable tmdl's.

\section{DNA/Genome Issues of Zoonotic Transfer}

Does the DNA of the recipient of mosquito saliva that is transmitted via biting become damaged causing the Zika virus results of deformed heads and neurological damage?

Even if the genome of the Aedes egyptii mosquito is normal, something in the saliva bite could effect the DNA/RNA genome of the recipient and thus the fetus.

Another theory might be that enzymes in the saliva of the mosquito affect the growth hormones of the developing fetus.

\section{Methods for Mosquito Abatement}

The following methods for mosquito abatement are recommended as outlined in an article in the Wall Street Journal

* "Battling "Cockroach of Mosquitoes"- Methods used to combat Zika mosquito- Sterilizing male mosquitoes with radiation.

- Genetically engineering mosquitoes

- Injecting mosquito eggs with Wolbachia bacteria

- Eliminating standing water

- Removing trash and water containers from homes

- Spraying insecticide inside and outside of homes (caution re strength and effects)

- Treating breeding spots with larvicide [7].

\section{Recommendations}

Organizations such as the UN with its sustainable and millennial development goals of improved housing, poverty eradication and universal health programs should include aggressive mosquito abatement programs as part of its development agenda [8]. Admirable efforts of conventions on safe use of mercury in mining and industry i.e., the Basel and the Minimata conventions need strong implementation and monitoring follow-up in policy recommendations. Agricultural biotechnology studies need to be funded that research the connections between crop production, pesticide use, transboundary movement of water and animals that may be contaminated both from agriculture and industry and mining, and the effect on drinking water especially in outlying areas and smaller cities. Biodiverse areas of the world need to be risk assessed for their contribution to health matters and climate change impacts [9]. The Zika forest and the Amazon river and forests hold many answers and challenges to human populations. The UN Convention on Biological Diversity and the resulting Aichi targets, adopted in Nagoya, Japan, in 2010, are notable, particularly Aichi Target 20 which recommends mobilizing financial resources to pay for ecosystem services and research at the local level. One idea is garnering revenues from parks and other tourism venues to fund more ecosystem services and studies i.e., watershed analyses and temperature studies.

\section{Summary}

The Zika virus has become a pandemic that must be documented as it spreads to assist health professionals in their pursuit of a vaccine as well as advising municipalities with mosquito abatement recommendations and other measures to protect the populace. Improving mosquito abatement practices at the local level should be paramount. With many choices of method as outlined in this article above, a reduction of the Aedes egyptii mosquito population should be achieved, if not complete eradication. That might just be too elusive and difficult to determinewhat's in your tool shed for mosquito abatement- it's a war out there!

\section{References}

1. UNICEF (2015) Web Article.

2. McKee PJ, MD.

3. Centers for Disease Control (Website Advisory)

4. Global Biodiversity (2016) ICLEI Cities Biodiversity Center.

5. Chemicals and Wastes Policy and Practice, IISD reporting services

6. Daniel MF (2015) Mountain Movers

7. Wall Street Journal, 2-12-2016, A7.

8. UN - Habitat SDG and MDG (goals) for post-2015.

9. Global Factors Driving Emerging Infectious Diseases, Wildlife Trust, NY, USA. 